\title{
A 900MHz CMOS Balanced Harmonic Mixer for Direct Conversion Receivers
}

\author{
Zhaofeng Zhang, Zhiheng Chen, Jack Lau \\ Department of Electrical \& Electronic Engineering \\ Hong Kong University of Science \& Technology \\ Clear Water Bay, Kowloon, Hong Kong \\ 852-2358-8843, 852-2358-1485(fax), eezzf@ee.ust.hk, eejlau@ee.ust.hk
}

\begin{abstract}
A $900 \mathrm{MHz}$ balanced harmonic mixer for direct conversion receivers is fabricated in a $0.35 \mu \mathrm{m}$ standard digital CMOS process. The self-mixing-induced DC offset is about $44 \mathrm{~dB}$ lower than that of the conventional mixer. The inputreferred offset is reduced to the noise level. Specific techniques on flicker noise reduction are also discussed. At $3 \mathrm{~V}$ power supply and $-15.4 \mathrm{dBm} L \mathrm{O}$ power, it achieves $13 \mathrm{~dB}$ conversion gain, $24.5 \mathrm{~dB}$ noise figure at $10 \mathrm{kHz},-10 \mathrm{dBm}$ third-order input intercept point and $+36 \mathrm{dBm}$ second-order input intercept point. The total power consumption is about $5 \mathrm{~mW}$.
\end{abstract}

\section{Introduction}

In recent years direct conversion architecture has gained much attention as a possible solution for a single-chip radio due to its low power, low complexity and easy-tointegrate properties. However, there are various design issues to be resolved, among which the DC offset generated by self-mixing and the flicker noise are the most critical. As most modulation schemes contain significant DC and low frequency components, base band offset cancellation is generally not a viable option, especially in the case of narrow band modulation. The bipolar balanced harmonic mixer proposed by $\mathrm{T}$. Yamaji[1] has the ability to reduce the offset down to the noise level. Nevertheless, no CMOS counterpart of this bipolar design has been reported in the literature. The dynamic matching technology proposed by E. Bautista[2] helps improve IIP2 and reduce flicker noise but the DC offset problem still exists. In this paper, a novel CMOS harmonic mixer with a unique working principle for the DC offset and flicker noise reduction will be introduced.

\section{Circuit Principle}

The self-mixing mechanism is illustrated in Fig. 1 where the LO leakage reflected back from the antenna, together with the amount of LO signal directly coupled to the input ports of the LNA and the mixer, is down converted to DC and becomes an offset. It is exacerbated when the reflection coefficient of the antenna varies with the environment.

The problem arises because the RF carrier and the LO signal are running at exactly the same frequency as shown in Fig. 2(a). For the harmonic mixing shown in

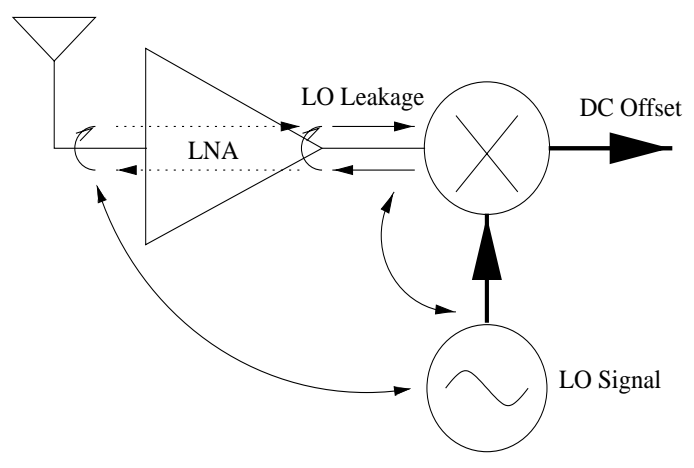

Figure 1 Self-mixing problem caused by LO leakage

Fig. 2(b), it is the second harmonic of the LO signal that takes part in the mixing process. As a result, the LO leakage generates no DC component but an output which is still situated at the LO frequency and can be easily filtered out.
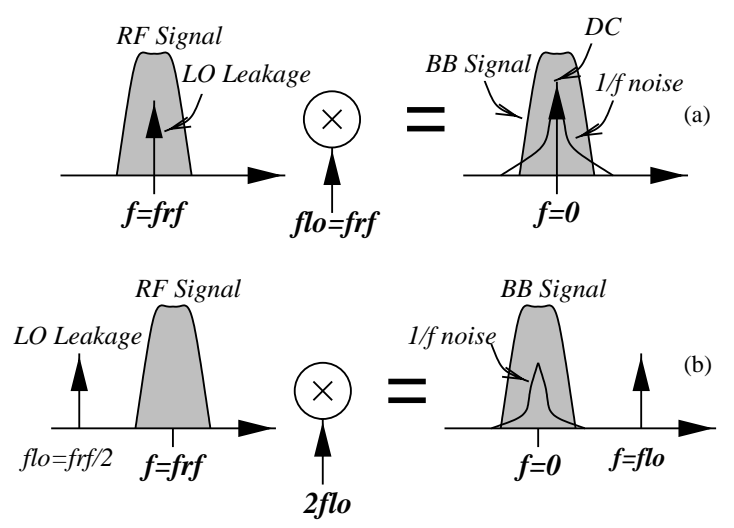

Figure 2 Working principles of (a) the conventional mixer and (b) the harmonic mixer

A CMOS balanced harmonic mixer which achieves a comparable performance to the bipolar version is shown 
in Fig. 3. The second harmonic is easily obtained because of the inherent square-law operation of the CMOS transistor. The LO stage is actually a squaring cell which converts the differential LO voltage to the time-varying current which contains the second harmonic. In principle, the fundamental and all odd harmonics of the LO will be cancelled out at the connected drain terminals and the DC offset problem will be mitigated.

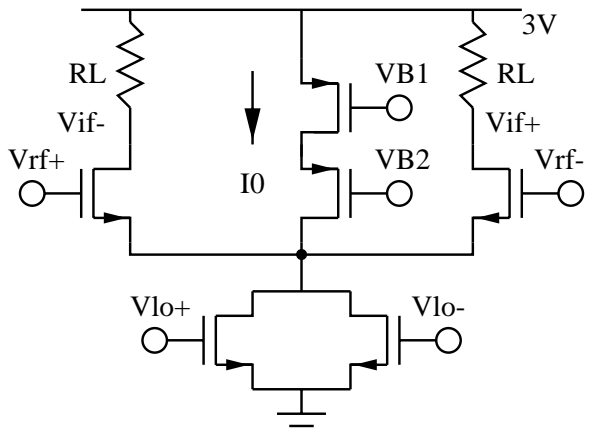

Figure 3 CMOS harmonic mixer with current injection

Flicker noise is another big problem in direct conversion receivers. Experimental results in Fig. 4 show that there is a minimum point for input-referred flicker noise, which is consistent with [3]. When the transistor size is increased, however, it is found that the optimal point moves towards the weak inversion region. To reduce the flicker noise, the RF part can be biased near this region. At a biasing current, a larger $\mathrm{W} / \mathrm{L}$ ratio drives the device toward the moderate or weak inversion region. This is very different from conventional mixers and offers the following advantages: the gain will be increased because transconductance increases with the $\mathrm{W} / \mathrm{L}$ ratio and the maximum value will be achieved in this region; the thermal noise will be decreased; the $1 / f$ noise will also decrease because of the large transistor size and it is near the optimal region; the inductive load of the LNA can be smaller; and $f_{\mathrm{T}}$ of the device will not degrade too much.

The objective of the injected current $I_{0}$ in Fig. 3 is to reduce the current in the two upper transistors driven by the RF signal. This helps reduce the $1 / f$ noise of the upper transistor pair, which is the main noise contributor. Besides the optimal flicker noise bias point, the noise reduction also comes from the increased ratio of $\mathrm{RF} / \mathrm{BB}$ gain to $\mathrm{BB} / \mathrm{BB}$ gain. The injected current itself will not introduce noise in this balanced structure. Unlike the normal Gilbert-type mixer, the two RF transistors change their currents simultaneously and any noise at their common source node will be completely cancelled out at the differential output. At the same time, the load of the mixer can be increased to raise the mixer

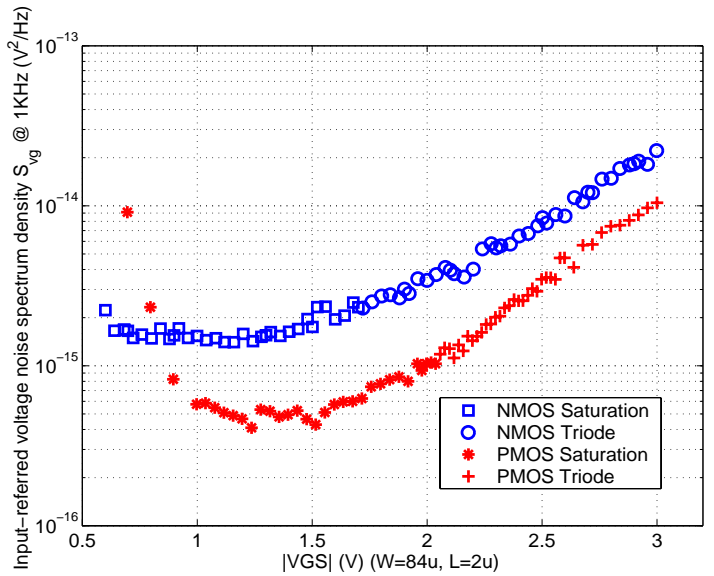

Figure 4 Measured input referred flicker noise versus bias

conversion gain. This method is also compatible with the low voltage application as there are only three stacked transistors. With the help of the injected current, more than $20 \mathrm{~dB}$ noise improvement can be achieved.

\section{Measurement and Discussion}

Fig. 5 gives the mixer measurement setup. A differential dynamic signal analyser, SR780, is used to measure the signal and noise spectrum. The low frequency buffer is fabricated together with the mixer on the chip and one individual buffer is also fabricated for deembedding and calibration.

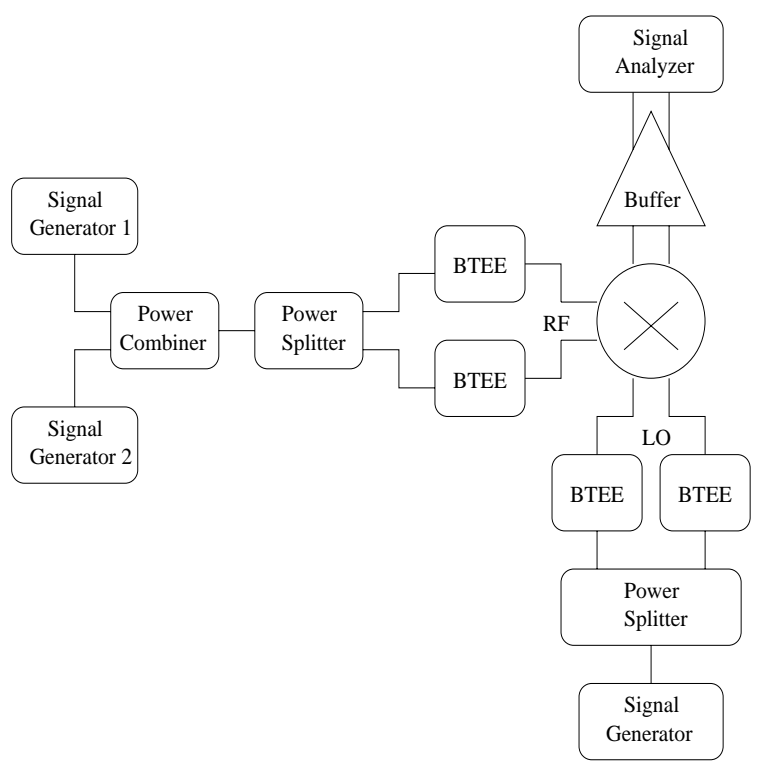

Figure 5 Mixer measurement setup

Fig. 6 shows the mixer conversion gain and offset cancellation ability. When measuring the LO leakage gain, a signal at the LO frequency rather than the RF frequency is used as the input. Ideally this gain is zero 
but there will be a finite value caused by on-chip device and off-chip component mismatch. When the LO power increases, the contribution of the device mismatch is less, and there is more improvement in the offset cancellation. Conversion gain also improves with LO input power due to increased current. At $-15.4 \mathrm{dBm} \mathrm{LO}$ power delivered to each side of the LO port, $37.5 \mathrm{~dB}$ offset cancellation is achieved. With the lower LO frequency, harmonic mixing also renders a higher LO to RF port isolation. Assuming a $20 \mathrm{~dB}$ per decade roll off, a $6 \mathrm{~dB}$ improvement is gained. With this advantage, the offset performance of our mixer will be $44 \mathrm{~dB}$ better than that of the conventional one. More improvement can be achieved with an integrated differential local oscillator. Assuming $50 \mathrm{~dB}$ LO to RF port isolation at $450 \mathrm{MHz}$, with $-15.4 \mathrm{dBm}$ LO input, the effective DC offset at the input of the mixer is about $-97 \mathrm{dBm}$, below the noise level at $200 \mathrm{kHz}$ noise bandwidth if LNA provides $20 \mathrm{~dB}$ gain and $5 \mathrm{~dB}$ noise figure.

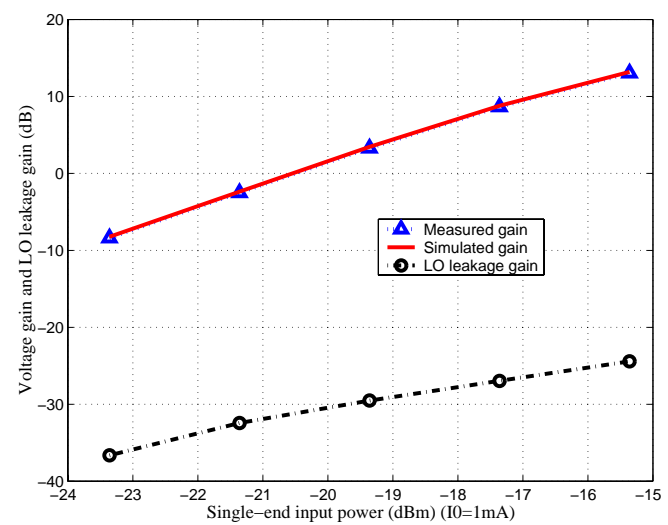

Figure 6 Offset cancellation (When measuring the LO leakage gain, a signal at the $L O$ frequency rather than that at the $R F$ frequency is used as the input.)

In Fig. 7 the conversion gain with respect to the injected current and LO power is plotted. The RF gain increases with the injected current until the device is pushed into the weak inversion where the square law relationship does not hold any longer. The higher the LO power, the larger the conversion gain.

However, the linearity degrades with the injected current, as shown in Fig. 8, due to the lowered effective gate voltage of the RF stage and smaller headroom with larger resistor load. When LO power increases, linearity also degrades. This occurs because the voltage drop on the resistor load increases with LO power and the headroom of the transistor becomes smaller. Therefore, there is a trade-off between conversion gain or noise and linearity. Fortunately, most receivers are using frequency or phase modulation and for this kind of system, usually the linearity requirement is not so

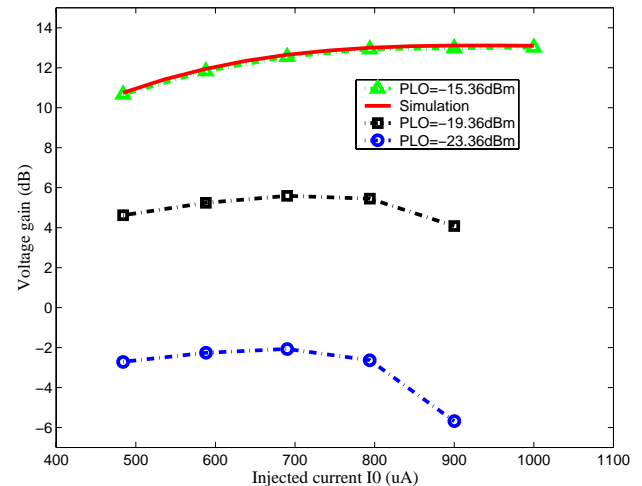

Figure 7 Measured conversion gain

stringent and some compromise can be made.

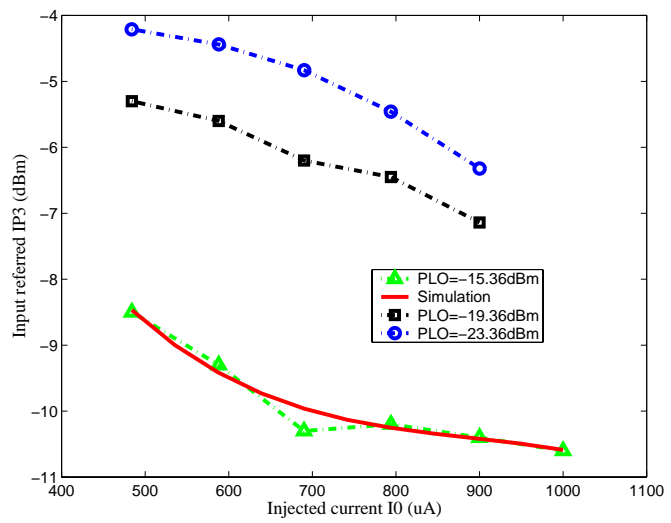

Figure 8 Measured input referred IIP3

The measured input-referred IP2 and IP3 under the best noise performance are shown in Fig. 9. IIP2 is important for direct conversion receivers. In addition to wellmatched devices, it can be further improved by adding some auxiliary circuits to compensate for the mismatch as done in [4].

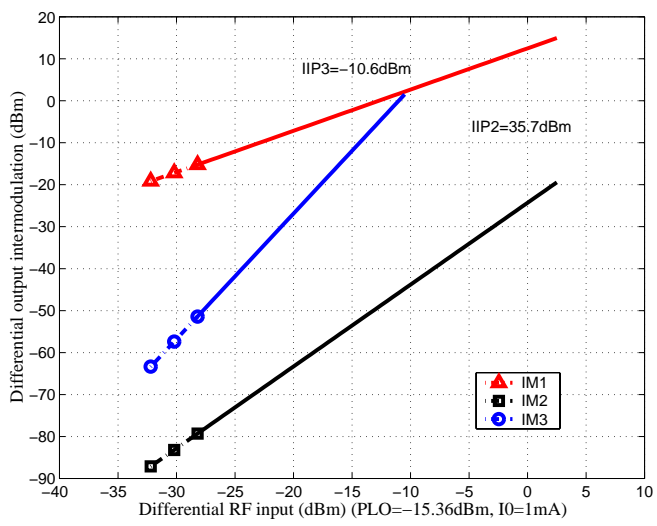

Figure 9 Measured IIP2 and IIP3 performance

In Fig. 10 the noise performance of the harmonic mixer is provided. With the current injection technique, the noise is greatly reduced. As expected, this is due to a 
lowered flicker noise level in the moderate inversion region and the increased RF signal gain to feed through gain ratio. Increased LO power also greatly improves noise performance because the conversion gain increases with the LO power. The output noise spectrum density is shown in Fig. 11. Flicker noise is obviously dominant.

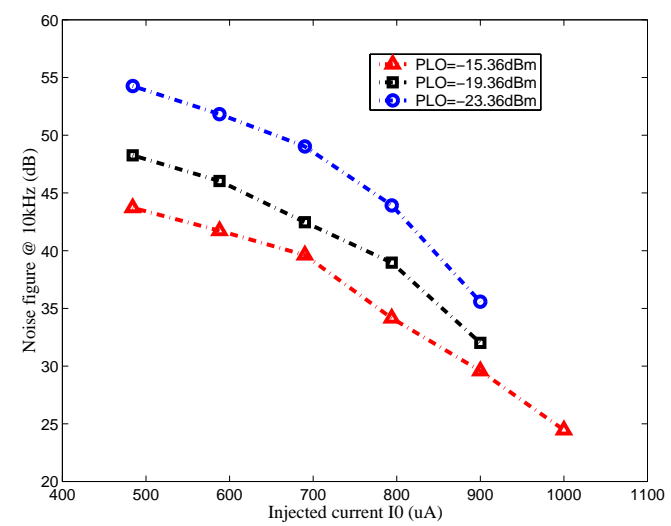

Figure 10 Measured noise figure versus injected current and LO input power.

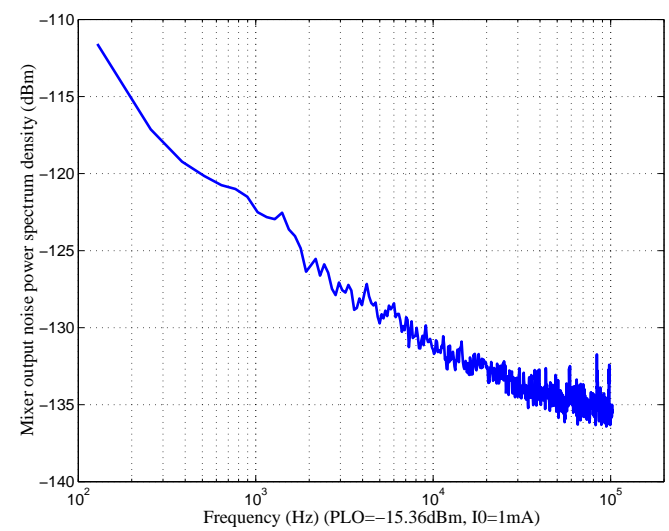

Figure 11 Mixer output noise spectrum density

The die photo of the CMOS harmonic mixer is shown in Fig. 12 and its performance is summarized in Table 1 .

\section{Conclusion}

In conclusion, the proposed CMOS harmonic mixer achieves the goal of DC offset free with the additional advantages of low complexity, low power consumption and low LO driving power. Two main problems in direct conversion receivers are alleviated. It is suitable for low cost highly integrated direct conversion receivers.

\section{Acknowledgments}

The authors would like to thank the UMAN group for their valuable discussions. The project was supported in part by RGC grant.

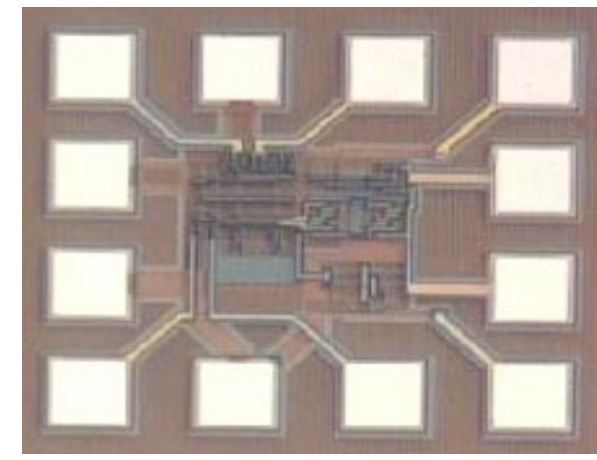

Figure 12 Die photo of the CMOS mixer

Table 1: Summary of Measured CMOS Harmonic Mixer

\begin{tabular}{|l|c|}
\hline Power supply & $3 \mathrm{~V}$ \\
\hline Single-ended LO power & $-15.4 \mathrm{dBm}$ \\
\hline LO frequency & $450 \mathrm{MHz}$ \\
\hline RF frequency & $900.05 \mathrm{MHz}$ \\
\hline Conversion gain & $13 \mathrm{~dB}$ \\
\hline 1dB compression point & $-19.9 \mathrm{dBm}$ \\
\hline IIP3 & $-10.6 \mathrm{dBm}$ \\
\hline IIP2 & $+35.7 \mathrm{dBm}$ \\
\hline Noise figure@ 10KHz & $24.5 \mathrm{~dB}$ \\
\hline DC offset cancellation & $37.5 \mathrm{~dB}$ \\
\hline DC current & $1.72 \mathrm{~mA}$ \\
\hline
\end{tabular}

\section{References}

[1] T. Yamaji, H. Tanimoto, "A 2GHz Balanced Harmonic Mixer for Direct-Conversion Receivers," Proc. of IEEE Custom IC Conf., pp9.6.1-9.6.4, May, 1997.

[2] E. Bautista, B. Bastani, J. Heck, "Improved Mixer IIP2 Through Dynamic Matching," ISSCC'2000, WP23.1, Feb., 2000.

[3] J. Chang, A. Abidi, C. Viswanathan, "Flicker Noise in CMOS Transistors from Subthreshold to Strong Inversion at Various Temperatures," IEEE Trans on Electron Devices, vol. 41, No. 11, pp.1965-1971, Nov., 1994.

[4] T. Yamaji, H.Tanimoto, H. Kokatsu, "A I/Q Active Balanced Harmonic Mixer with IM2 Cancelers and a $45^{\circ}$ Phase Shifter," IEEE Journal of Solid-State Circuits, vol. 33, No. 12, pp.2240-2246, Dec., 1998. 